\title{
TOPICAL CORTICOSTEROIDS IN ORAL PATHOLOGY
}

Adriana Krasteva ${ }^{1}$, Assya Krasteva ${ }^{2}$, Angelina Kisselova ${ }^{2}$

${ }^{I}$ Department of Pharmacology and Toxicology, Faculty of Pharmacy,

${ }^{2}$ Department of Imaging and Oral Diagnostic, Faculty of Dental Medicine, Medical University, Sofia, Bulgaria

\section{ABSTRACT}

Topical corticosteroids have been commonly used over the last 50 years to treat various inflammatory mucosal conditions. The wide spectrum of potencies and bases allows these medications to be used effectively and safely under the care of an experienced dental practitioner.

\section{INTRODUCTION:}

Topical corticosteroids are some of the most common drugs used in oral pathology for treating atrophicerosive lesions that affect the mucosa. These lesions often bleed and are painful; sometimes are chronic or have a high tendency to reappear and to interfere with very important activities such as eating, drinking, speaking or associating with other people. Severe erosive lesions of the oral mucosa have classically been treated with systemic corticosteroids, although the adverse effects associated with the use of these drugs have conditioned the frequent prescription of topical corticosteroids for treating these pathologies.

Some of the most common TCs are presented in favour of dental practitioner when choosing the most appropriate therapy to each clinical case.

Table 1. Topical corticosterois classified by potency

\begin{tabular}{|c|c|c|}
\hline Steroids & $\begin{array}{l}\text { Posology in } \\
\text { every } 6 \text { hours }\end{array}$ & Comments \\
\hline \multicolumn{3}{|l|}{ WITH MODERATE EFFECT } \\
\hline - Betamethasone valerate $0,05 \%$ cream (Betnovate) & $\begin{array}{l}\text { To be applied on the } \\
\text { lesion }\end{array}$ & $\begin{array}{l}\text { growth or adrenal suppression is } \\
\text { possible }\end{array}$ \\
\hline Mometasone $0,1 \%$ cream (Elocom) & $\begin{array}{l}\text { To be applied on the } \\
\text { lesion }\end{array}$ & $\begin{array}{l}\text { growth or adrenal suppression is } \\
\text { possible }\end{array}$ \\
\hline - Triamcinolone acetonide $0,1 \%$ cream (Polocortolon) & $\begin{array}{l}\text { To be applied on the } \\
\text { lesion }\end{array}$ & $\begin{array}{l}\text { growth or adrenal suppression is } \\
\text { possible }\end{array}$ \\
\hline \multicolumn{3}{|l|}{ WITH POTENT EFFECT } \\
\hline $\begin{array}{l}\text { Beclomethasone dipropionate spray(Beconase, Becotide } \\
\text { inhaler, Ecobec inhaler) }\end{array}$ & $\begin{array}{l}1 \text { press of the pump, } \\
100 \mu \mathrm{m}\end{array}$ & $\begin{array}{l}\text { growth or adrenal suppression is } \\
\text { possible }\end{array}$ \\
\hline $\begin{array}{l}\text { Budenoside spray(Budenoside forte, Neo-Reactive, } \\
\text { Pulmicort turbuhaler) }\end{array}$ & $\begin{array}{l}1 \text { press of the pump, } \\
100 \mu \mathrm{m}\end{array}$ & $\begin{array}{l}\text { growth or adrenal suppression is } \\
\text { possible }\end{array}$ \\
\hline - Fluticasone $0,05 \%$ cream (Cutivate) & $\begin{array}{l}\text { To be applied on the } \\
\text { lesion }\end{array}$ & $\begin{array}{l}\text { growth or adrenal suppression is } \\
\text { possible }\end{array}$ \\
\hline \multicolumn{3}{|l|}{ WITH VERY POTENT EFFECT } \\
\hline $\begin{array}{l}\text { Betamethasone dipropionate \& Gentamycin } 0,05 \% \text { cream, } \\
\text { ointment (Diprogenta) }\end{array}$ & $\begin{array}{l}\text { To be applied on the } \\
\text { lesion }\end{array}$ & $\begin{array}{l}\text { growth or adrenal suppression is } \\
\text { possible }\end{array}$ \\
\hline $\begin{array}{l}\text { Clobetasol propionate } 0,05 \% \text { cream, ointment (Clobederm, } \\
\text { Dermovate) }\end{array}$ & $\begin{array}{l}\text { To be applied on the } \\
\text { lesion }\end{array}$ & $\begin{array}{l}\text { growth or adrenal suppression is } \\
\text { possible }\end{array}$ \\
\hline
\end{tabular}


Table 2. Some topical steroids

\begin{tabular}{|l|l|l|}
\hline \multicolumn{1}{|c|}{ MILD POTENCY } & \multicolumn{1}{|c|}{ MODERATE POTENCY } & \multicolumn{1}{c|}{ HIGH POTENCY* } \\
\hline $\begin{array}{l}\text { Triamcinolone acetonide 0,1\% cream, } \\
\text { ointment (Polcortolon) }\end{array}$ & Fluocinonide 0,5\% cream (Metosyn) & $\begin{array}{l}\text { Clobetasol propionate 0,5\% cream, } \\
\text { ointment(Clobederm, Dermovate) }\end{array}$ \\
$\begin{array}{l}\text { Fluocinolone acetonide 0,025\% } \\
\text { cream, ointment (Abricort, Flucinar, } \\
\text { Synalar) }\end{array}$ & $\begin{array}{l}\text { Desoximetasone } 0,25 \% \quad \text { cream } \\
\text { (Stiedex) }\end{array}$ & $\begin{array}{l}\text { Betamethasone valerate 0,05\% cream, } \\
\text { ointment (Betnovate, Dermocort, } \\
\text { Diprosone) }\end{array}$ \\
$\begin{array}{l}\text { Betamethasone valerate 0,05\% cream } \\
\text { Betnovate) }\end{array}$ & & \\
\hline Fluticasone $0,05 \%$ cream (Cutivate)
\end{tabular}

*Sterois with high potency should be avoided in patients with gastroduodenal ulcers, diabetes mellitus, hematological malignancies, hepatitis, pregnancy and brestfeeding.

Short-term use of systemic corticosteroids is generally well tolerated, but the risk of adverse events increases with duration of use. The side effects from 3-week courses are few and mild, but such courses may only be done once every 3 months.

Table 3. Systemic steroids dose tapering

\begin{tabular}{|l|c|c|c|c|c|c|c|c|c|c|c|c|cc|}
\hline Day & 1 & 3 & 5 & 7 & 9 & 11 & 13 & 15 & 17 & 19 & 21 & 23 & 25 & 27 \\
\hline Number tablets after bearkfast & 12 & 12 & 12 & 9 & 9 & 9 & 9 & 6 & 6 & 6 & 6 & 3 & 2 & 1 \\
\hline $\mathrm{mg} / \mathrm{d}$ & 60 & 60 & 60 & 45 & 45 & 45 & 45 & 30 & 30 & 30 & 30 & 15 & 10 & 5 \\
\hline
\end{tabular}

Short-term treatment: 110 tablets prednisolone $5 \mathrm{mg}$, 4-weeks period.

\section{REFERENCES:}

1. I. Hargitai, R. Sherman, Corticosteroids in Dentistry. Clinical Update. Naval Postgraduate Dental School National Naval Dental Center Bethesda, Maryland 2001;23:11-13.
2. N. Savage, MJ McCullough. Topical corticosteroids in dental practice. Aust Dent J 2005; 2:40-44.

\section{Address for correspondence:}

Adriana Krasteva

Department of Pharmacology and Toxicology, Faculty of Pharmacy,

Medical University, Sofia, Bulgaria

E-mail: adriakr@gmail.com 